\title{
Identification of adverse reactions to new drugs. IV-Verification of suspected adverse reactions
}

\author{
GEOFFREY R VENNING
}

\begin{abstract}
The verification processes were assessed for $18 \mathrm{important}$ adverse drug reactions. Verification when achieved by formal studies was not obtained through cohort studies such as postmarketing surveillance or other follow up of drug users but through case-control studies or a similar disease orientated approach. When not achieved in this way the most useful aspects of uncontrolled studies were rechallenge and dose-response data.

Analysis of the data and of the characteristics of different methods of verification suggested that there were four approaches to earlier discovery. (1) The best system seemed to be some form of record linkage, capable of providing at the same time data on incidence of adverse reactions and on prevalence of drug usage in patients with disease suspected of being drug induced. Until such a system can be established the relative efficacy of alternative approaches appears to be (2) review of all published first alerts, with prompt initiation of case-control or disease orientated studies for verification, (3) postmarketing surveillance of cohorts of drug users, and (4) voluntary reporting systems.
\end{abstract}

\section{Introduction}

In part I of this series ${ }^{1} 18$ important adverse reactions to new drugs were identified. These were reviewed in part $\mathrm{II}^{2}{ }^{3}$ and in part $\mathrm{III}^{4}$ they were analysed in terms of alerting processes and early-warning systems. The following study concludes the series by examining how the suspected adverse reactions were verified.

\section{Verification}

Table I shows the study designs and type of evidence providing convincing verification. Of the 18 adverse reactions, 15 were type $B$ and three type A. ${ }^{5}$ One of the 18 reactions required no verification. Causality was established at the first alerting stage for phenformin and lactic acidosis 10 years before marketing. The evidence was experimental. This is a type A reaction. A causal relation was not, however, self evident in the case of two other suspected adverse reactions, both of delayed onset and now classifiable as type A-namely, stilboestrol and vaginal cancer, and phenothiazines and tardive dyskinesia. Six further adverse reactions were verified by formally designed

University Department of Community Medicine and General Practice, Oxford

GEOFFREY R VENNING, BM, FRCP, visiting scientist (now director of research and development, Janssen Pharmaceutical Limited, Marlow, Bucks SL7 1ET) studies. In every case the design was based on a survey of patients with the disease observed, and in no case was the verification dependent on a clinical trial or cohort study.

Proponents of the cohort approach, and of postmarketing surveillance schemes in particular, might argue that large scale postmarketing studies were not carried out and might have been effective in identifying these adverse reactions. Against this, however, it may be pointed out that large scale studies were indeed carried out with five drugs responsible for eight of the 18 adverse reactions under review (oral contraceptives, practolol, stilboestrol, halothane, phenothiazines); of the remaining 10 , the reaction to phenformin was identified at the clinical trial stage, leaving nine where one might speculate on the possible utility of postmarketing surveillance schemes including about 10000 patients. These would almost certainly have failed to identify eight of the nine reactions, whose incidence was very low-the three pseudomembranous colitis reactions, deaths from asthma, phenacetin nephropathy, aplastic anaemia associated with phenylbutazone and chloramphenicol, and clioquinol subacute myelo-optic neuropathy. The only one where a 10000 sample might have been adequate was methysergide retroperitoneal fibrosis; in this case an incidence of 2 in 500 was reported in the original alert. If the study was representative the adverse reaction could have been detected at the clinical trial stage without the need for postmarketing surveillance.

It is difficult to avoid the conclusion that the case orientated approach is always likely to be more efficient than the cohort approach for type $\mathrm{B}$ reactions, both being obviously more reliable than voluntary reporting systems. So far as the six adverse reactions verified by case orientated studies were concerned, two reports were case-control

TABLE I-Study designs and type of evidence providing verification of 18 important adverse drug reactions

\begin{tabular}{|c|c|c|c|}
\hline Adverse reaction & Drug & $\begin{array}{l}\text { Type of evidence providing } \\
\text { convincing verification }\end{array}$ & Type* \\
\hline \multicolumn{4}{|c|}{ Cohort study (with data on incidence of adverse reaction) } \\
\hline \multicolumn{3}{|c|}{$\begin{array}{l}\text { Valid case studies (with data on prevalence of drug } \\
\text { usage in patients with adverse reaction) }\end{array}$} & A \\
\hline Thromboembolism & Oral contraceptives & $\begin{array}{l}\text { Control data-case control } \\
\text { study; also, later, dose- } \\
\text { response data }\end{array}$ & $\mathbf{B}$ \\
\hline Vaginal cancer & Stilboestrol & Control data-case control & A \\
\hline Deaths from asthma & Aerosols & Control data-case control & B \\
\hline Aplastic anaemia & Phenylbutazone & $\begin{array}{l}\text { Data on prevalence of drug } \\
\text { usage }\end{array}$ & B \\
\hline Pseudomembranous colitis & Lincomycin & Data on prevalence of drug & B \\
\hline Aplastic anaemia & Chloramphenicol & $\begin{array}{l}\text { usage } \\
\text { Data on prevalence of drug } \\
\text { usage }\end{array}$ & B \\
\hline \multicolumn{4}{|c|}{ Other studies including anecdotal reports } \\
\hline Myocardial infarction & Oral contraceptives & Dose-response data & B \\
\hline Jaundice & Halothane & Rechallenge data & $\mathbb{B}_{\mathbf{B}}$ \\
\hline Rash & Practolol & Rechallenge data & $\begin{array}{l}\mathbf{B} \\
\mathbf{B}\end{array}$ \\
\hline $\begin{array}{l}\text { Colitis } \\
\text { Sclerosing peritonitis }\end{array}$ & $\begin{array}{l}\text { Antibiotic } \\
\text { Practolol }\end{array}$ & $\begin{array}{l}\text { Rechallenge data } \\
\text { Rarity of event }\end{array}$ & B \\
\hline Tardive dyskinesia & Phenothiazines & Rarity of event & \\
\hline Colitis & & Rarity of event & \\
\hline $\begin{array}{l}\text { Retroperitoneal fibrosis } \\
\text { Keratoconjunctivitis }\end{array}$ & Methysergide & Rarity of event & $\begin{array}{l}\mathbf{B} \\
\mathbf{B}\end{array}$ \\
\hline $\begin{array}{l}\text { Keratoconjunctivitis } \\
\text { Subacute myelo-optic } \\
\text { neuropathy }\end{array}$ & $\begin{array}{l}\text { Practolol } \\
\text { Clioquinol }\end{array}$ & $\begin{array}{l}\text { Special clinical teatures } \\
\text { No convincing verification }\end{array}$ & $\mathbf{B}$ \\
\hline
\end{tabular}

*Rawlins and Thompson's classification." 
studies and four others were valid case studies giving data on prevalence of drug usage among patients with the suspected reaction. In each of these latter reports the evidence was convincing without the need for a control group. These studies, however, used the same fundamental approach as the case-control study, and it may therefore be important to emphasise that for planned verification of an important suspected adverse reaction the case study approach should normally be considered first in preference to the cohort approach. Finally, it should not be forgotten that both alerting and verification of the thalidomide phocomelia problem were also dependent on the case orientated approach.

Discussions on the subject conventionally point out the theoretical deficiencies of case-control studies and emphasise the need for the cohort approach. In an analysis of the relative importance of cohort and case-control studies Jick ${ }^{6}$ recognised the problem but regarded the cohort method as the research approach of choice for newly marketed drugs, with the case reference approach as appropriate only for marketed drugs. He did not break the problem down further in terms of alerting and verification. In a later publication Jick et $a l^{7}$ discussed "systems to obtain additional information on adverse drug effects after marketing." For the subset of unrecognised effects they concluded that "the methodological approach required is the follow up study" and that, "because uncommon effects are a major concern large cohorts are required for each drug of interest."

Lawson and Wilson ${ }^{8}$ also reviewed the pros and cons of what they described as drug orientated and disease and complication orientated systems. Although they did not reach definite conclusions on the preferred approach, they cited a wider range of adverse drug reactions identified by the latter, including phocomelia due to thalidomide, smoking and lung cancer, aminorex and pulmonary hypertension, phenytoin and congenital malformations, and several others. It is clear, however, from the data presented here that large postmarketing surveillance schemes as envisaged in numerous publications are likely to be far less efficient for the prompt verification of suspected adverse reactions than systems based on case finding, whether formal casecontrol studies or other valid case studies. It may therefore be important to emphasise that postmarketing surveillance schemes will need to be justified almost entirely on the basis of their potential contribution to alerting. Alternative schemes capable in addition of making a useful contribution to verification of suspected adverse reactions would therefore be more useful.

The verification mechanisms that operated in the remaining 11 instances need discussion. For two adverse reactions dose-response data were important. Dose-response data also contributed to later confirmation of the causal relation between oral contraceptives and thromboembolism. (The stage at which this was identified as "verified" is a personal opinion; this particular issue, however, was the subject of controversy at the time.) Phenacetin nephropathy was verified on this basis in a cross sectional prevalence study, which is a logical formal design that does not strictly fit into the classification chosen in this survey. The oral contraceptive myocardial infarction reaction was verified by detection of a dose-response relation in a survey of reports on adverse reactions whose design did not permit any estimate of either incidence of the adverse reaction or of prevalence of drug usage among patients with the event. The lesson to be learnt from this experience may be that in the absence of satisfactory data from cohort or valid case studies careful assessment of dose-response data may often be critical. Put another way, when faced with lack of information on the denominator it is important to look for dose-response data during analysis of the numerator. In comparison with the formal case-control studies which confirmed the verification of the oral contraceptive myocardial infarction reaction and extended our knowledge about other contributory factors the original numerator analysis approach was bound to save several years.

For three adverse reactions verification was dependent on rechallenge data. In two cases this was fortuitous (halothane jaundice and tetracycline pseudomembranous colitis) but in the other was deliberate (practolol dermatitis). There may well be many types of adverse reactions where a deliberate and cautious rechallenge experiment would be ethically justifiable; it is beneficial to the patient to know whether a drug chosen for its efficacy may still be available; greater attention to rechallenge where justifiable may result in satisfactory verification much more promptly than is happening at present. For practolol keratoconjunctivitis verification was dependent on a combination of several factors. The existence of eye lesions with unusual clinical features was noted early; when several cases were observed in which these lesions were associated with a rash already verified as a side effect of practolol there was no reasonable doubt about causality.

For four adverse reactions where there is now no reasonable doubt about validity the moment of verification was difficult to identify precisely. These are reactions where the observed clinical event is rare in the absence of the drug and apparently much commoner in drug users, but data on incidence among users and of prevalence of drug usage among patients with the reaction have never been satisfactorily established. This applies to sclerosing peritonitis with practolol, colitis with clindamycin, retroperitoneal fibrosis with methysergide (the reported figure based on two events being unacceptable as evidence of incidence), and tardive dyskinesia with phenothiazines. The remaining reaction is clioquinol/subacute myelo-optic neuropathy. As pointed out in part II, the evidence for verification has been challenged, but despite this many would agree that the regulatory action was prudent, and the problem cannot now be further resolved.

As with alerting there has been little or no contribution from national voluntary reporting systems, the British yellow cards, phase IV clinical trials, postmarketing surveillance schemes, or hospital surveillance programmes such as the Boston Collaborative Drug Surveillance Program. These mechanisms contributed to verification of only two adverse reactions. Inman's study ${ }^{9}$ of deaths from myocardial infarction associated with oral contraceptives was based on yellow card reports supplemented by similar voluntary adverse reaction reports in Denmark and Sweden. It should be noted that the reporting systems did not actually provide verification, which came from a specific study in which a dose-response relation was identified.

Yellow card reporting also contributed to the verification of the practolol peritonitis reaction, whose date cannot be fixed precisely. In considering the role of national voluntary reporting systems an inherent possible disadvantage cannot be entirely discounted. These systems have discouraged physicians from reporting adverse reactions directly to manufacturers. Despite this, the adverse drug reaction files kept by companies may have contributed almost as much as the national systems to the discovery of these reactions, or perhaps the national schemes contributed almost as little as the pre-existing manufacturers' systems. The verification of phenylbutazone aplastic anaemia was partly dependent on the company adverse reaction files, and the manufacturers of practolol invited physicians to report skin and eye problems after the first alerts. This could have contributed to subsequent verification of the oculomucocutaneous syndrome, although the actual verification of the eye problem arose from further work by the investigator responsible for the first alert.

While this study shows that voluntary reporting systems contributed very little to the first alerting and to the verification of these important adverse reactions, this does not mean that such systems have no utility - merely that other systems are likely to be more effective for these defined purposes. A fair assessment of voluntary reporting systems should include other factors than those considered here. Between first alerting and verification they may provide additional information, and after verification they give information on frequency of reporting. Continuing information of the patterns of reporting adverse reactions may be of help to the Committee on the Safety of Medicines and to the Department of Health and Social Security in developing policies for the licensing of new drugs and the review of product licences of right for old drugs in various therapeutic categories.

Though yellow cards provide an unsatisfactory data base for casecontrol studies, there may be occasions when an unsatisfactory data base is better than none at all. Table II summarises the contributions of the various systems to verification.

\section{Incidence of adverse reactions}

In assessing the feasibility of detecting adverse reactions by different approaches information on the distribution of actual incidence among different important reactions is obviously critical. In reviewing the problem, Jick ${ }^{6}$ regarded an incidence of 1 in 200 or greater as high and of 1 in 10000 or less as low, with others as intermediate. The choice of these levels of demarcation, though arbitrary, is very logical. Adverse reactions with an incidence of 1 in 200 or greater could be identified at the clinical trial stage, where data are usually available on about 1000 patients before a product licence is granted. Adverse reactions with an intermediate frequency of $<1$ in 200 or $>1$ in 10000 might be identified in postmarketing surveillance schemes, whereas those of low frequency $-<1$ in $10000-$ may be verified only in case-control studies. It is therefore of considerable importance to assess the incidence of these adverse reactions. We need to know the overall incidence in users of the drug and also where possible in subsets of users-for example, those receiving high dosage, long duration of treatment, repeated administration of drugs normally given once only, and in other subsets where relevant to the occurrence of the adverse reaction. 
TABLE II-Contributions to alerting and verification of 18 adverse drug reactions from established systems

\begin{tabular}{|c|c|c|c|}
\hline & First alert* & $\begin{array}{l}\text { Contributed to } \\
\text { later verification }\end{array}$ & $\begin{array}{l}\text { Verification } \\
\text { dependent on } \\
\text { system }\end{array}$ \\
\hline $\begin{array}{l}\text { National voluntary } \\
\text { reporting systems }\end{array}$ & $\begin{array}{l}\text { 1/18. Aerosol } \\
\text { deaths, } \\
\text { Australia }\end{array}$ & $\begin{array}{l}\text { 1/18. Practolol } \\
\text { peritonitis }\end{array}$ & $\begin{array}{l}\text { 1/18. Orai } \\
\text { contraceptives, } \\
\text { myocardial } \\
\text { infarction }\end{array}$ \\
\hline $\begin{array}{l}\text { Company systems, phase } \\
\text { IV trials, and adverse } \\
\text { drug reaction files }\end{array}$ & $0 / 18$ & $\begin{array}{l}\text { 2/18. Practolol/ } \\
\text { keratitis; } \\
\text { phenylbutazone } \\
\text { aplastic anaemia }\end{array}$ & $0 / 18$ \\
\hline $\begin{array}{l}\text { Hospital surveillance } \\
\text { schemes (for example, } \\
\text { Boston programme) }\end{array}$ & $0 / 18$ & $0 / 18$ & $0 / 18$ \\
\hline $\begin{array}{l}\text { Monitoring of national } \\
\text { mortality statistics }\end{array}$ & $0 / 18$ & $\begin{array}{l}\text { 1/18. Aerosol } \\
\text { deaths, UK }\end{array}$ & $\begin{array}{l}\text { 1/18. Decline in } \\
\text { aerosol deaths } \\
\text { after warning }\end{array}$ \\
\hline
\end{tabular}

*Yellow card system began in 1964 and Boston Collaborative Drug Surveillance Program in 1966. Alerts for some reactions had already occurred.

To assess total incidence the number of adverse reaction reports on yellow cards may be of some limited value for those drugs mainly used by general practitioners, where the denominator of usage is available for recent years. Inman and Vessey, however, pointed out that the incidence of yellow card reports was as low as less than $1 \%$ or as high as $15 \%$ of the true incidence for different reactions. ${ }^{10}$ The extent of underreporting is not uniform and may be subject to bias when there is publicity concerning a suspected adverse reaction. Warnings sent to the medical profession by the Committee on the Safety of Medicines clearly contribute to such publicity and could therefore create bias and render subsequent yellow card data misleading. There may be circumstances where this could hinder or delay the verification of a suspected adverse drug reaction. For fatal reactions yellow card reports are supplemented by data from death certificates provided by the Office of Population Censuses and Surveys, and the extent of underreporting is likely to be less.

Review of the data on incidence makes it possible to place many of these adverse reactions into the categories defined by $\mathrm{Jick}^{6}$ for consideration of appropriate strategies for detection. Out of 18 adverse reactions, two were inappropriate for this assessmentsubacute myelo-optic neuropathy as it was not satisfactorily defined or verified, and phenacetin nephropathy as it is an abuse phenomenon. Of the remaining 16, no fewer than 13 fell in the category of low incidence with the drug and low background incidence. Jick proposed that a cohort approach was desirable for this category for newly marketed drugs. From the data discussed in part II it seems that this would almost certainly have been ineffective for these serious adverse reactions unless cohort sizes in excess of 100000 users had been followed up, including long term observation in some instances. A case orientated approach was effective in several instances and could have been in several more. The three adverse reactions with a higher incidence were the three type A reactions where samples of the size usually available at the clinical trial stage are theoretically adequate for detection. In the case of phenformin lactic acidosis this in fact occurred; in another-stilboestrol and vaginal cancer-the long time factor was critical in preventing this and a case-control approach was effective. The third-tardive dyskinesia from phenothiazines-is also a delayed variant of a reaction that was identified at the clinical trial stage.

It may therefore be stated that for these 18 important adverse reactions three type $A$ reactions were, in theory, detectable at the clinical trial stage and 12 of 15 type B reactions were of such low incidence that they were unlikely to be detectable by cohort based postmarketing surveillance schemes. The other three type $B$ reactions also had a low incidence of the fully developed important clinical syndrome, but minor variations of the reaction were detectable at the clinical trial stage-skin and eye lesions with practolol, and liver function abnormalities in the subset of repeated halothane anaesthetics. Analysis in terms of incidence categories thus fully supports the thesis that the detection of important adverse drug reactions requires two approaches: improved monitoring and event recording at the clinical trial stage, and prompt initiation of case-control or other case orientated studies as soon as a first alert occurs. Record linkage schemes should be effective as they make possible a case orientated analysis in addition to identifying cohorts of drug users.

The contribution of cohort approaches after marketing seems unlikely to be important. The clear superiority of case orientated approaches and the obvious defects of voluntary reporting systems suggest that a case orientated registry approach deserves consideration, particularly for the very low incidence haematological reactions, as identified in part II.

\section{Conclusions}

In reviewing the features critical for verification of suspected adverse reactions to drugs dose-response data and rechallenge data were important. These features were lacking in the earliere studies of these suspected reactions. They may therefore be important considerations in the design of clinical trials of new⿳亠丷⿵冂丶 drugs.

One of the lessons from the halothane story concerns routine음 monitoring for safety during clinical trials. At present regulatory agencies demand that such monitoring should include laboratory tests for renal, hepatic, and haematological adverse reactions as』 well as clinical monitoring and any additional tests indicated by the particular toxic effects noted in animal studies. The late. confirmation of hepatic toxicity with halothane came from a $\vec{\omega}$ controlled study on a subset of patients receiving repeated $\omega$ anaesthetics. The question of monitoring adequate numbers of $\frac{}{3}$ patients in various subsets and of monitoring control groups may need further consideration.

\section{LESSONS FOR POSTMARKETING PHASE}

Reviewing the time of verification of suspected adverse reactions showed that delays occurred in seven instances. In two of these the eventual verification was dependent on rechallenge; $\vec{N}$ deliberate with practolol dermatitis, and fortuitous with halo- 7 thane jaundice. In the other five instances the successful studies leading to verification were all case studies or case-control studies. In every instance verification would have taken place $₹$ sooner if the studies had been initiated sooner. In one case the $\vec{\varphi}$ delay was two years, in three cases five years, and in one case $\mathscr{C}^{\infty}$ nine years. The essential requirements for initiating a case- $\square$ control study promptly seem to be the following: (1) someone needs to identify the need for studies of this type after first alerts; (2) someone needs to set aside funds for the purpose; and (3) investigators need access to a source of patients with the $\frac{\mathbb{Q}}{\varnothing}$ disease which is suspected of being drug induced; for rare $\varrho$ diseases some form of registry is needed. It is important that the $\overrightarrow{\overrightarrow{0}}$ registry should not be biased.

The yellow card reports may have helped with eventual verification of practolol peritonitis (but made no contribution to first alerting or verification of dermatitis and keratoconjunctivitis). Yellow card reports also provided a data base for the verification of the oral contraceptive myocardial infarction reaction. This 0 was, however, dependent on the emergence of a dose-response 3 . relation, and many people were convinced only when a proper $\delta$ case-control study was carried out later; yellow card data do not provide a suitable basis for case-control studies.

\section{RELATIVE EFFICACY OF EARLY-WARNING SYSTEMS}

As was noted earlier, the ideal system for the identification of $\mathcal{O}$ adverse drug reactions would be a record linkage system, $\omega$ providing at the same time data on the incidence of reactions among drug users and the prevalence of usage of a variety ofo drugs among patients with diseases and syndromes that might be $\Phi$ drug induced. Until such a system is operating on a regular basis $?$ there are three main approaches possible to the identification of $\underline{7}$ adverse reactions; (1) systematic initiation of disease or case $\frac{O}{\mathbb{D}}$ orientated studies as soon as a first alert occurs-case-control $\stackrel{\mathbb{\Phi}}{\stackrel{\Phi}{\$}}$ studies are preferable but require a data base of cases from an $\stackrel{\mathbb{Q}}{\circ}$ unbiased registry or similar source; (2) drug orientated studies, $\overline{2}$ such as postmarketing surveillance schemes which give data on 8 incidence; and (3) voluntary reporting systems which fail to응 provide prevalence data and give rise to biased registries often unsuitable for verification. The data from the present survey 
indicate that the first approach is the most useful and the second approach is preferable to the third.

The successful formal verification of adverse drug reactions is often dependent on individuals who use the first approach. Assessment of the voluntary reporting systems cannot, however, be made solely on the basis of the factors reviewed here without consideration of other ways in which they may have proved useful.

\section{Overall summary and conclusions of series}

Many important adverse reactions are pharmacologically unpredictable, with an incidence of one in 10000 or less. This has important implications for the sample size needed in postmarketing surveillance studies entailing follow up of a cohort of drug users. Unless sample sizes of 100000 are accepted as practicable such cohort studies will be useful only for identifying more frequent reactions. More information is badly needed on the incidence of serious reactions, such as colitis with lincomycin and clindamycin.

Delays to alerting are a minor problem compared with delays before verification and regulatory action. Alerting usually occurs through published anecdotal reports. Voluntary reporting and other "early warning systems" have made a negligible contribution to alerting. Event recording, if combined with the use of patients as their own controls, might lead to identification at the clinical trial stage of minor adverse reactions, thus alerting to the risk of more serious reactions in the same organ system. Routine monitoring for drug safety should include patients in various subsets where there may be a particular liability to adverse reactions.

The possibility of false alerts makes verification essential. Dose-response data and rechallenge are both important for this purpose. Additional anecdotal reports do not necessarily constitute valid verification, depending on the extent of drug usage and the frequency of the observed disease in non-users. The possibility has been noted of false verification through bias in reporting after regulatory warnings or other publicity. When formal verification has been obtained of suspected serious reactions this has been through surveys of patients with particular diseases and not through follow up of drug user cohorts. There are compelling arguments for case orientated rather than drug orientated verification studies. This approach may not have been used by regulatory authorities because it is counterintuitive, but the intuitive approach entailing postmarketing surveillance of cohorts of drug users has been unproductive and is likely to be of limited value for reasons stated. Schemes including record linkage, however, include case analysis as well as drug user analysis and are better for this and other reasons.

Analysis of disease patterns by specialists in different disciplines of medicine might contribute more to early discovery of serious reactions than drug surveillance. Both for alerting and for verification there is a need for registries of the types of disease that are commonly drug induced-for example, blood dyscrasias. Unbiased registries are necessary to provide data suitable for case-control studies. Voluntary adverse reaction reports are inappropriate for this purpose.

I thank Sir Richard Doll, Professor Abe Goldberg, Dr G Jones, Dr David Skegg, and Professor Martin Vessey for helpful comments and criticisms of the analysis, and Professor D J Weatherall for advice on presentation of this series of papers.

\section{References}

1 Venning GR. Identification of adverse reactions to new drugs. I-What have been the important adverse reactions since thalidomide? $\mathrm{Br}$ Med $\mathcal{F}$ $1983 ; 286: 199-202$.

2 Venning GR. Identification of adverse reactions to new drugs. II-How were 18 important adverse reactions discovered and with what delays ? Br Med F 1983;286:289-92.

3 Venning GR. Identification of adverse reactions to new drugs. II (continued)-How were 18 important adverse reactions discovered and with what delays? Br Med $\mathcal{7} 1983 ; \mathbf{2 8 6}: 365-8$

4 Venning GR. Identification of adverse reactions to new drugs. IIIAlerting processes and early-warning systems. Br Med $\mathcal{F} 1983 ; \mathbf{2 8 6}$ 458-60.

${ }^{5}$ Rawlins MD, Thompson JW. Pathogenesis of adverse drug reactions. In Davies DM, ed. Textbook of adverse drug reactions. Oxford: Oxford University Press, 1981.

${ }^{6}$ Jick H. The discovery of drug induced illness. N Engl f Med 1977;296 481-5.

7 Jick H, Walker AM, Spriet-Pourra C. Post-marketing follow-up. FAMA $1979 ; 242: 2310-4$.

"Lawson DH, Wilson GM. Detecting adverse drug reactions. Br $\mathcal{F}$ Hosp Med 1974;12:790-8.

${ }^{9}$ Inman WHW, Vessey MP, Westerholm B, Engelund A. Thromboembolic disease and the steroidal content of oral contraceptives; a report to the Committee on Safety of Drugs. Br Med F 1970;ii:203-9.

10 Inman WHW, Vessey MP. Monitoring adverse drug reactions. In: Bennett AE, ed. Recent advances in community medicine. Edinburgh: Churchill Livingstone, 1978.

(Accepted 11 November 1982)
I recently saw a 60 year old woman with Bell's palsy who told me that 12 days previously she had spent eight hours with a friend in Scotland. The friend had been diagnosed as having Bell's palsy five days before my patient arrived. The two friends kissed each other goodbye. Was this merely a coincidence, or have other cases of apparent transmission of Bell's palsy been recorded?

Idiopathic facial palsy, or Bell's palsy as it is more popularly known, is, as its title implies, of unknown aetiology. The three favoured theories are ischaemia produced by exposure to cold-for example, sitting in a draught - a virus infection, or a variant of the allergic neuritis that can be produced by antigen-antibody reactions in man and in animal studies. ${ }^{1}$ The infectious aetiology is supported by the association of facial palsy with known viral infections such as varicella, mumps, herpes zoster, and the EB virus (infectious mononucleosis), ${ }^{2}$ and in such instances transmission could conceivably result from kissing. Further support for the virus infectious aetiology comes from the report of clustering of cases in Israel, in some instances almost amounting to epidemics. ${ }^{2}$ Against the theory of an infectious aetiology in the case described is the age of the patient as older patients are thought more likely to have a vascular cause for Bell's palsy. Nevertheless, although I am not aware of any reported cases of apparent transmission of Bell's palsy, on the evidence given I think that this was more than a coincidence, and the two cases probably lend support to the infection theory of Bell's palsy. $-N$ E F CARTLIDGE, consultant neurologist and senior lecturer in neurology, Newcastle upon Tyne.

' Miller H. Facial paralysis. Br Med f 1967;ii:815-9.

Leibowitz U. Epidemic incidence of Bell's palsy. Brain 1969;92:109-14

Can lithium treatment be used for a patient who suffers from manic depressive psychosis and hypothyroidism?

As goitre and hypothyroidism are not uncommon side effects of lithium treatment, it is an established routine to monitor thyroid function before lithium treatment is started and periodically thereafter. Should lithium induced hypothyroidism develop, it may be treated with concurrent thyroxine. Pre-existing hypothyroidism treated with thyroxine is therefore not an absolute contraindication to using lithium for the treatment of manic depressive psychosis, although the indications for its use should be assessed even more carefully than usual, given that lithium treatment is more effective in the prophylaxis of bipolar than unipolar affective disorders. If episodes of manic depressive psychosis in a hypothyroid patient are severe and disabling lithium treatment might be started when the patient is euthyroid, having been stabilised with thyroxine maintenance. Rigorous clinical and biochemical monitoring of thyroid function is obligatory.-A HAMID GHODSE, consultant psychiatrist, London. 